\title{
284. Atypische Hemipelvektomie bei schwersten Kombinationsverletzungen des Beckens
}

\author{
H. Thiele, R. O. Bethke ${ }^{1}$, M. M. Linder, U. Bethke ${ }^{2}$
}

Chirurgische Klinik, ${ }^{1}$ Unfallchirurgische Klinik, und ${ }^{2}$ Institut für Anästhesiologie und Intensivmedizin des Klinikums Mannheim der Universität Heidelberg, Theodor-Kutzer-Ufer, D-6800 Mannheim

\section{Atypical Hemipelvectomy in Combined Pelvic Injuries}

Summary. A total of 24 pelvic fractures combined with abdominal, visceral, genito-urinary tract and vascular injuries were treated during a 10-year period (1 January 1973 to 31 December 1982). Type of accident: road injury (12), crush injury (7), fall from high altitude (3), buried alive (2). Group I: trauma with vascular lesion (13), mortality $57 \%$; group II : without vascular lesion (9), mortality $12 \%$. Two cases of major crush injury of the pelvis are reported. Both patients survived after atypical hemipelvectomy.

Key words: Pelvic fracture - Vascular lesion - Bowel injury - Hemipelvectomy.

Zusammenfassung. Vom 1. 1. 1973 bis 31. 12. 1982 wurden 24 schwere Beckenkombinationstraumen behandelt. Ursachen : Verkehrsunfall $n=12$, Quetschverletzung $n=7$, Verschüttung $n=2$, Sturz aus großer Höhe $n=3$. Gruppe I: mit Verletzung von Iliacal- bzw. Femoralgefäßen, Letalität: $57 \%(n=15)$. Gruppe II: ohne Gefäßbeteiligung, Letalität: $12 \%(n=9)$. Zwei Fälle von schwerer Quetschverletzung (einmal durch Eisenbahnpuffer, einmal durch Papierwalzen werden geschildert). Beide Verletzte überlebten nach atypischer Hemipelvektomie.

Schliisselwörter: Beckenfraktur - Gefäßverletzung - Darmverletzung - Hemipelvektomie.

\section{Der kindliche Oberschenkelbruch - eine Domäne der konservativen Knochenbruchbehandlung}

\author{
L.-J. Lugger, J. Oberhammer und H. Resch \\ Universitätsklinik für Unfallchirurgie (Vorstand i. V. Univ.-Doz. Dr. L.-J. Lugger), \\ Anichstraße 35, A-6020 Innsbruck
}

\section{The Femoral Fracture in Children - a Domain of Conservative Treatment}

Summary. The "uncomplicated" femoral fracture in children should still be treated conservatively. Large areas of soft-tissue interposition, widely dislocated and rotated fragments, the very proximal shaft fracture, and extremely long hospitalization during schooltime are all relative indications for operative treatment. A permanent rotational deformity of more than $10^{\circ}$ in $20-30 \%$ of the fractures demanded no osteosynthesis. Based on a study of 122 conservatively treated childhood femoral fractures and a followed-up group of 55 , it was found that rotational deformities were spontaneously and steadily corrected without peaks at age 7 and 12 . The use of sonography to control alignment and fragment position reduces irradiation exposure by one-third.

Key words: Infant femoral fracture - Late results - Rotational deformity - Sonography.

Zusammenfassung. Der ,unkomplizierte“ Oberschenkelbruch ist eine Domäne konservativer Frakturbehandlung geblieben. Große Weichteilinterposition, Fragmentdislokation und -rotation, die sehr proximale Schaftfraktur und überlanger Spitalaufenthalt im Schulalter stellen relative Operationsindikationen dar. Die in $20-30 \%$ verbleibende Rotationsfehlstellung von mehr als $10^{\circ}$ ist keine Operationsindikation. Eine Vorstudie an 122 konservativ behandelten kindlichen Oberschenkelbrüchen und eine weitere Untersuchung von 55 Fällen zeigt ein kontinuierliches spontanes Rotationskorrekturverhalten ohne „Detorsionsschub“. Sonographische Achsenmessungen können die Strahlenbelastung um ein Drittel reduzieren.

Schlüisselwörter: Oberschenkelbruch, kindlich - Spätergebnisse - Rotationsfehlstellung - Sonographische Achsenmessung. 